\title{
Post-wrinkle bifurcations in elastic bilayers with modest contrast in modulus
}

\author{
Anesia Auguste ${ }^{1}$, Lihua Jin ${ }^{2}$, Zhigang Suo ${ }^{2}$ and Ryan C. Hayward ${ }^{1}$
}

1 Department of Polymer Science \& Engineering, University of Massachusetts, Amherst, MA, 01003, USA.*E-mail: hayward@umass.edu

2 School of Engineering and Applied Sciences, Kavli Institute for Nanobio Science and Technology, Harvard University, Cambridge, MA, 02138, USA.*E-mail: suo@ seas.harvard.edu

\begin{abstract}
Wrinkles, folds, creases and other elastic surface instabilities play a crucial role in many systems in nature and engineering. While surface instabilities of ideal bilayer structures with large contrasts in elastic stiffness are well understood, many natural and man-made structures are far from this ideal. To better understand the behavior of systems with modest stiffness contrast, in particular their secondary post-wrinkling bifurcations, we systematically vary the modulus contrast between the film and the substrate through a combination of experiments and finite element simulations. Above a modulus contrast of about 2, but below approximately 14, wrinkles represent the primary bifurcation mode, but can undergo two distinct types of secondary bifurcations upon further compression: (1) a direct transition from wrinkles to creases, and (2) wrinkles that first undergo period doubling, followed by a transition to creases.
\end{abstract}

\section{Introduction}

Surface buckling instabilities are observed in everyday life, from wrinkles on skin to folds in the brain. Such processes arise when an elastic material is compressed beyond a critical strain either by swelling, differential growth, or mechanical forces. In engineering, similar instabilities have long been thought of as nuisances, but they have recently found applications in many different contexts including flexible electronic devices[1-4], surface patterning methods[5-8] and materials with tunable optical properties[9-12], adhesion[13-15], and wettability[9,16,17].

In biological systems, many tissues and organs are composed of multilayers with similar moduli, which may undergo a variety of buckling instabilities during growth or deformation.[1823] Thus, understanding the buckling behavior of multilayers with a modest contrast in elastic modulus may provide insight into pattern formation in such systems. However, most studies have focused on bilayers with a much stiffer thin film on top of a compliant thick substrate,[24- 
26] and thus there remains a lack of understanding of surface instabilities when the ratio of the film to substrate modulus is modest, i.e., from $\sim 2$ to 10 .

In previous studies[27,28], we and others have examined the primary bifurcation, i.e., the first instability mode to appear in an elastic bilayer upon compression from the flat state, and found that either creases or wrinkles can occur first, depending on the ratio of the moduli and thicknesses of the layers. A stiffer and thinner film tends to form smooth wrinkles under small compressive strains, while a softer and thicker film tends to form self-contacting creases[29-32] under larger strains. For a bilayer with a sufficiently large contrast in modulus and thickness contrast, wrinkles result from a competition between the stretching energy of the substrate and the bending energy of the film. Above a critical compressive strain, wrinkles appear with an initial wavelength set by the film thickness and the modulus ratio of the film to the substrate. Upon further compression of a wrinkled bilayer, secondary bifurcations occur. If the substrate is not highly pre-stretched, period doubling - wherein the amplitude of every other wrinkle peak grows while its neighbors shrink - is the next mode to appear,[33-37] followed by period quadrupling and eventually folding.

Near the boundary between where wrinkles and creases compete as the primary bifurcation mode, however, there is a region where wrinkles can transition into creases with further compression. In this region, the modulus ratio between the film and the substrate is modest. There are relatively few studies on elastic bilayers with modest modulus contrast[28,3842]. Hutchinson and coworkers showed computationally that at low modulus contrast, wrinkles can transition into creases, though their simulations were not continued beyond this point[40]. Tallinen, et al., performed a computational study of how the difference in stiffness between two layers influences the transition from wrinkles to creases or folds upon growth of the film relative to the substrate [42]. Kim and coworkers[43] also showed that wrinkles can transition to creases for graded samples with an elastic stiffness that decreases with distance from the free surface. Later, they studied the evolution of the surface instabilities in bilayers with low modulus ratio using computational methods and constructed a phase diagram of three qualitatively different types of post-wrinkling bifurcations[39]: (i) wrinkles that transition directly to creases, (ii) wrinkles that undergo period doubling and then form creases, and (iii) wrinkles that undergo period doubling, period quadrupling and then folding. However, this study was solely 
computational. Wang, et al. computationally identified three different post-wrinkle bifurcation modes: period-doubling, creasing, and delamination buckling, and experimentally showed the existence of each $[28,41]$. However, their simulations could not continue past the onset of creases. In addition, both studies reported by Wang, et al.[28,41], and Tallinen, et al. [42], involve differential pre-stretch of the substrate compared to the film, which is known to have important effects on post-wrinkling behavior[37,44,45].

In this paper, we combine experiments and simulations to track the post-wrinkle bifurcations of elastic bilayers with a modest modulus ratio between 2 and 10. Our work builds upon the finding of Kim and coworkers with regards to two qualitatively different types of secondary bifurcations from wrinkles to creases.[39] In particular, we determine the conditions for coexistence between different modes of instability, the presence or absence of hysteresis in the transition from wrinkles to creases, and the preferred spacing between post-wrinkle creases, all in samples with no substrate pre-stretch

\section{Methods}

\section{Finite element simulations}

We conduct two-dimensional finite element simulations using the commercial software ABAQUS to study the initiation and evolution of surface instability modes, especially the secondary bifurcations, in elastic bilayers with modest modulus contrast. Such elastomeric materials with strains under 0.5 are commonly described by the neo-Hookean model, which has led to good agreement with experiments in previous work $[37,39,41,46]$, thus we model both the film and substrate as incompressible neo-Hookean materials, with perfect bonding between the layers. We set the total thickness of the bilayer to be 50 times the film thickness so that the substrate is effectively infinite. We first use a linear perturbation analysis to determine the wavelength of wrinkles in the bilayers, $L_{w}$. Then we perform a second simulation to capture the quasi-static process of the wrinkle formation and the transition of several wrinkles to one crease. By varying the size of the simulation box, we can vary the spacing of creases $L_{c}$.

To break the translational symmetry of the surface, we introduce the first eigenmode from the linear perturbation analysis with a small amplitude value $\left(5 \times 10^{-4}\right.$ times the film 
thickness) into the second simulation as an initial imperfection of the system. Besides that, a geometric defect is also introduced on the surface of the film to set the location where a crease will form, and also ensure the formation of only one crease in the simulation box. The size of the geometric defect is $5 \times 10^{-3}$ times the film thickness to otherwise minimize the effect of the defect. On the other hand, the mesh is refined around the geometric defect to resolve the field. We assume the crease to be symmetric, and thus apply a mirror symmetry condition on one side of the simulation box, with the geometric defect located at the corner of that boundary and the top surface of the film. On the other side of the simulation box, homogeneous compression is applied. The Riks method is used to solve the boundary value problem so that not only the stable solutions but also the unstable solutions can be captured. An extremely compliant elastic layer (with modulus $10^{-2}$ times that of the film) is introduced on top of the film to facilitate the convergence of the simulation when contact of the crease is involved, as we have used and tested in our previous studies[27,47,46]. Moreover, to realize the uniaxial compression condition as in the experiment by a 2D model, we simulate an axisymmetric ring under uniaxial compression in the direction of the symmetry axis, as employed in our previous work [46]. The radius of the ring is set to be 10 times of the total thickness of the bilayers so that the effect of the curvature of the ring is small. Element type $\mathrm{CAX} 8 \mathrm{H}$ is used.

\section{Experiment}

To study post-wrinkle bifurcation experimentally, we use a method similar to that reported previously (Fig. 1)[27]. An elastic 'mounting layer', comprising a silica reinforced vinyl polydimethylsiloxane with a shear modulus of $260 \mathrm{kPa}$ (DMS-V31S15, Gelest), is pre-stretched using a custom built stretcher. The polydimethylsiloxane (Sylgard 184) substrate (300-500 $\mu \mathrm{m}$ thick) is pre-cured in a petri dish for $16 \mathrm{~h}$ at $40{ }^{\circ} \mathrm{C}$, then cut to length $L$ and attached to the mounting layer by using a small amount of uncured polydimethylsiloxane of the same composition as the substrate as an adhesive layer. A glass slide is treated with a vapor phase of trimethylchlorosilane (Gelest) for $2 \mathrm{~h}$ at room temperature. Next, the film is spin coated onto the glass slide and partially cured at $120^{\circ} \mathrm{C}$ for $10 \mathrm{~min}$. The partially cured film is attached to the

substrate and placed in an oven at $40{ }^{\circ} \mathrm{C}$ for $16 \mathrm{~h}$. This two-step curing process provides a strong bond between the film and substrate without the use of an adhesive. The crosslink density of 
Sylgard 184 is varied for the film and the substrate by using compositions of 5:1, 7.5:1, 10:1, 20:1, and 30:1 (weight ratio of base to cross-linker) to access a range of moduli (2.6 $\pm 0.8 \mathrm{MPa}$, $2.1 \pm 0.5 \mathrm{MPa}, 1.6 \pm 0.5 \mathrm{MPa}, 0.75 \pm 0.08 \mathrm{MPa}$ and $0.4 \pm 0.05 \mathrm{MPa}$, respectively)[48,49]. Although we use these values (measured for homogeneous layers of each composition) to define the nominal modulus contrast, we note that the two-step curing process used here to prepare bilayers can lead to systematic deviations from these values (e.g., due to migration of polymer and/or crosslinker from the film into the substrate), thus making direct quantitative comparisons of experiments and simulations at the same modulus ratio difficult. The film thickness was held constant at $18 \pm 3 \mu \mathrm{m}$, except in the experiment to study the effect of film thickness on crease spacing, where it was varied from 4 to $26 \mu \mathrm{m}$ by altering the spincoating speed. Once the bilayer is fully cured, the mounting layer is released to a length $l$, adding compressive strain $\varepsilon=(L-$ $l) / L$, to the film and substrate in small increments of $0.02-0.03$, with at least 10 min between subsequent steps to minimize the influence of viscoelastic dissipation..

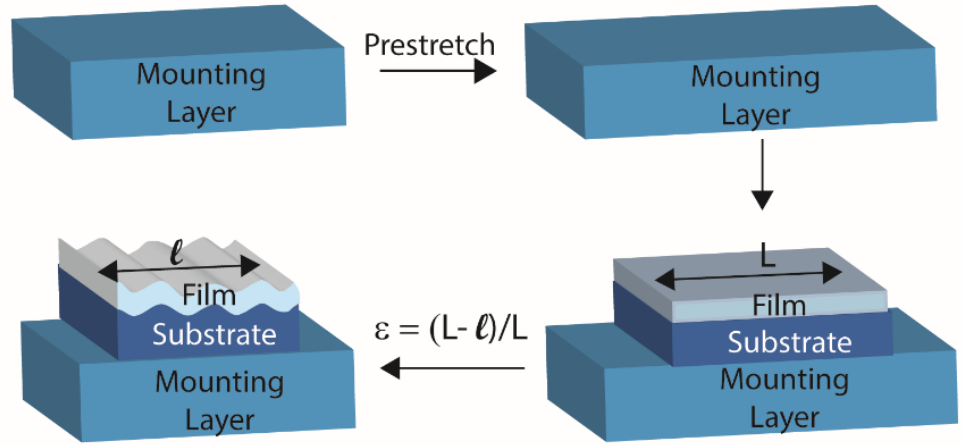

Fig. 1. Schematic illustration of experiments on compressed bilayers. The mounting layer is first pre-stretched. On top of the mounting layer, the bilayer (i.e., the substrate and film) is attached at no strain and at initial length $L$. The mounting layer is released to apply a compressive strain $\varepsilon$ to the bilayer.

\section{Results}

\section{Type 1 post-wrinkle bifurcation}

Kim and co-workers have predicted that, at a modulus ratio between 1.74 and 5.86, as the compressive strain increases, wrinkles will transition directly into creases[39]. We call this type of transition a 'type 1' post-wrinkle bifurcation. To further elucidate the nature of this 
bifurcation, we perform finite element simulations of the initiation and evolution of the surface instability for a bilayer with $G_{f} / G_{s}=3$, where $G_{f}$ is the shear modulus of the film and $G_{s}$ the shear modulus of the substrate. In Fig. 2a, we plot the bifurcation diagram with the external strain $\varepsilon$ as the loading parameter, and the energy normalized by the value of the flat state $U / U_{0}$ as the state of the field. When the strain is small, the flat state is the only solution. When the strain is large enough, the solutions of wrinkle and crease start to emerge. The insets of Fig. 2a show the simulation results of the wrinkle state and the crease state. Wrinkles will first form on the flat surface at strain $\varepsilon=0.30$. However, the energy of the crease solution will soon be lower than the wrinkle solution. At the Maxwell condition, the wrinkle state and the crease state have the same energy. The wrinkle state becomes unstable at $\varepsilon=0.309$.

With the emergence of the crease solution, wrinkles lose their periodicity. When the crease spacing is set to $L_{c}=3 L_{w}$, we distinguish two different types of wrinkle troughs, one of which transitions to a crease and the other which does not. Fig. $2 \mathrm{~b}$ shows the bifurcation diagram with the amplitude normalized by the film thickness $H_{f}$ as the state of the field. The amplitude is defined as half of the vertical distance between one wrinkle trough and the neighboring wrinkle peak, as sketched in the inset of Fig. 2b. Especially, here we calculate the amplitudes of the two different troughs and the wrinkle peak between them; we mark the curves for the two different types of troughs as 'crease tip' and 'wrinkle trough' respectively in Fig. 2b. The amplitude remains zero until the initiation of wrinkles. After the emergence of the crease solution, the two curves bifurcate from one another. The crease tip grows deeper and therefore the amplitude gets larger, while the other amplitude gets smaller and eventually approaches zero. 

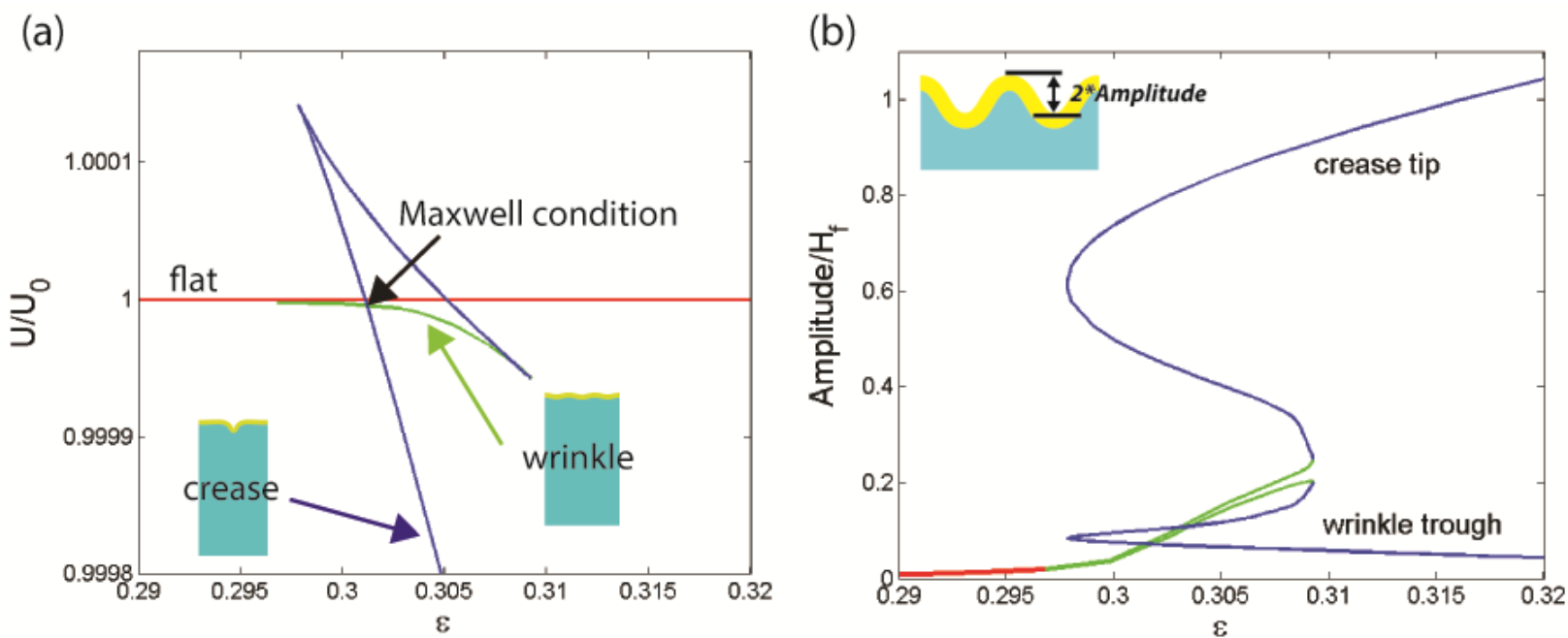

Fig. 2. Type 1 post-wrinkle bifurcation: wrinkles transition directly to creases $\left(G_{f} / G_{s}=3\right)$. In both bifurcation diagrams, the loading parameter is the uniaxial strain $\varepsilon$. (a) The state of the field is represented by the free energy of the block normalized by the flat state $U / U_{0}$. The insets show the simulation results of the wrinkle state and the crease state. (b) The state of the field is represented by the amplitude normalized by the film thickness $H_{f}$.

Experiments demonstrate that wrinkles transition directly to creases in an elastic bilayer with a modulus contrast below 5, in agreement with the simulation. The sample in Fig. 3a has a modulus contrast of approximately $4(1.6 \pm 0.5 \mathrm{MPa}$ film and $0.40 \pm 0.050 \mathrm{MPa}$ substrate). At a film strain of 0.28, faint wrinkles first form (Fig. 3a). As compression is increased to 0.29, creases nucleate in some of the trough of the wrinkles. Once creases nucleate, they channel across the surface and relax the wrinkles to zero amplitude over a period of 10 min, leaving behind only the creases.

Confocal microscopy images show the cross-section of a sample with a modulus contrast of approximately $3.5(750 \pm 80 \mathrm{kPa}$ substrate and $2.6 \pm 0.080 \mathrm{MPa}$ film $)$ going through a type 1 post-wrinkle bifurcation during compressive loading and unloading (Fig. 3b). Fluorescein oacrylate is added to the film to image the cross-section of the film. Faint wrinkles appear at a strain of 0.33 , representing a small increase in the critical strain compared to the sample in Fig. 3a, consistent with the slightly reduced modulus contrast in Fig. 3b. As the compression increases, the wrinkles snap to a localized crease with a finite depth and the neighboring region flattened. Further compression leads to the creases growing in depth. When the compression is subsequently decreased, the crease depth decreases until the system snaps directly back to the 
flat state, bypassing the wrinkle state, and clearly demonstrating the hysteretic nature of the subcritical type 1 wrinkle-to-crease transition.
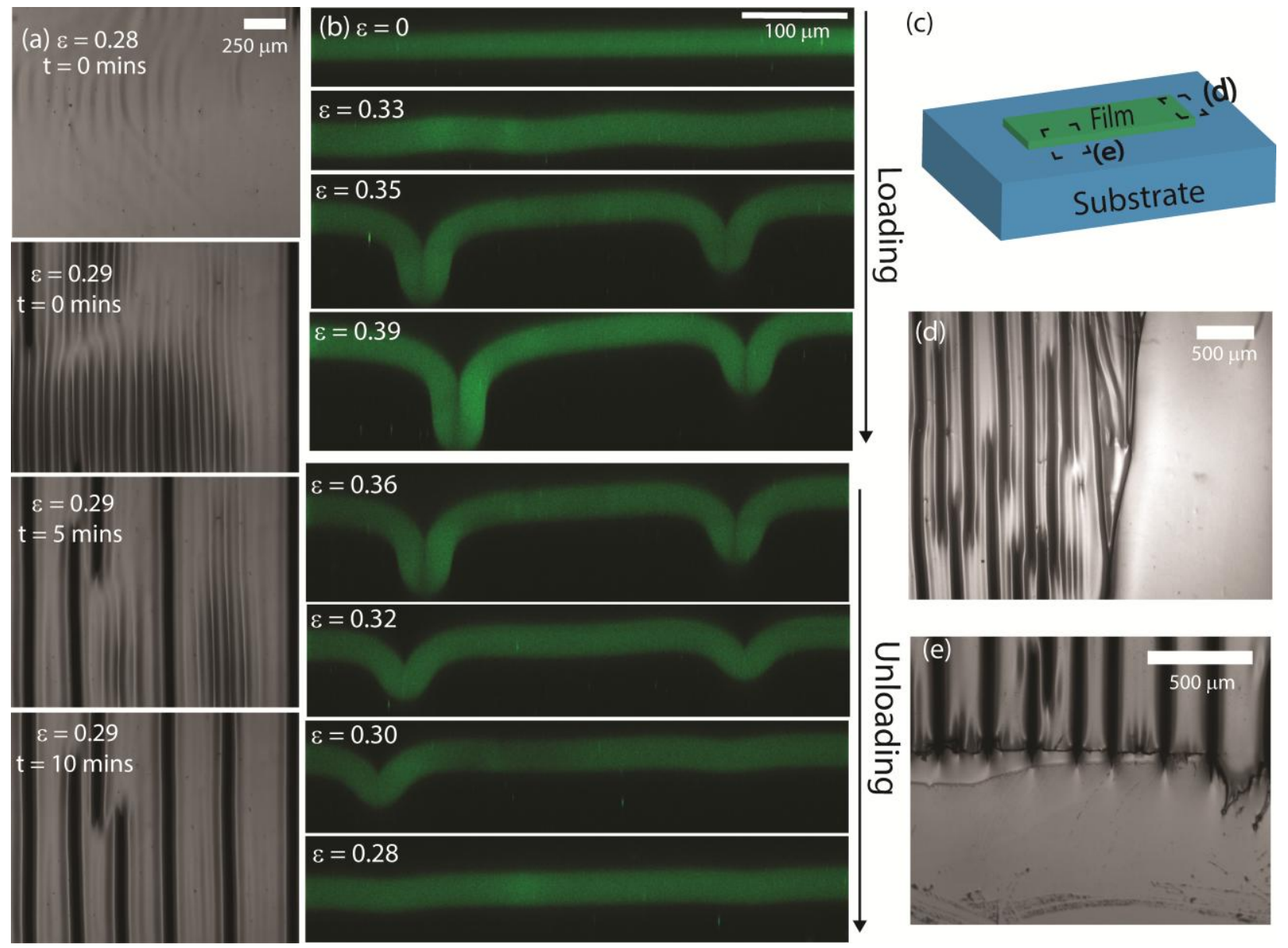

Fig. 3. Experimental observations of type 1 post-wrinkle bifurcation. (a) Optical micrographs show that, as the compressive strain increases, wrinkles first appear, and then creases nucleate and channel in the troughs of the wrinkles $\left(G_{\mathrm{f}} / G_{\mathrm{s}} \approx 4\right)$. (b) Confocal image of the cross section of a bilayer during loading and unloading $\left(G_{\mathrm{f}} / G_{\mathrm{s}} \approx 3.5\right)$. The florescent dye is present in the film, but not in the substrate. (c) Schematic of a strip of film partially covering the substrate, with indications of the location of images (d) and (e). (d) For a sample with a film strain of 0.33 , the right half of the optical micrograph shows the bare substrate and the left half is the film attached to the substrate, which shows the coexistence of wrinkles and creases. (e) Creases do not propagate onto the bare substrate below a film strain of 0.44 . 
In another experiment, a small strip of the film covers a portion of the substrate, while the surrounding areas of the substrate are left bare (Fig. 3c). We then examine the edges of the film, as denoted by the two dotted boxes in Fig. $3 \mathrm{c}$ and shown in Fig. 3d and 3e. The left part of the Fig. 3d image is the substrate covered by a film with a modulus contrast of 4 while the right part does not have the film. Similarly, the top part of the image in Fig. 3e is covered with the film while bottom part is not and the bare substrate is exposed. These images demonstrate two important points. First, the presence of a slightly stiffer film significantly lowers the critical strain for creases compared to a homogeneous material like the bare substrate. This is evident in both Fig. 3d and e, where creases are present in the bilayer but the bare substrate remains flat at a strain of 0.33. Secondly, the creases will remain on the bilayer and will not channel into the regions where the film is not present as shown in Fig. 3e. Once the system is compressed beyond the critical strain of 0.44 for creasing in a thick soft substrate under uniaxial compression, creases are formed on the bare substrate.
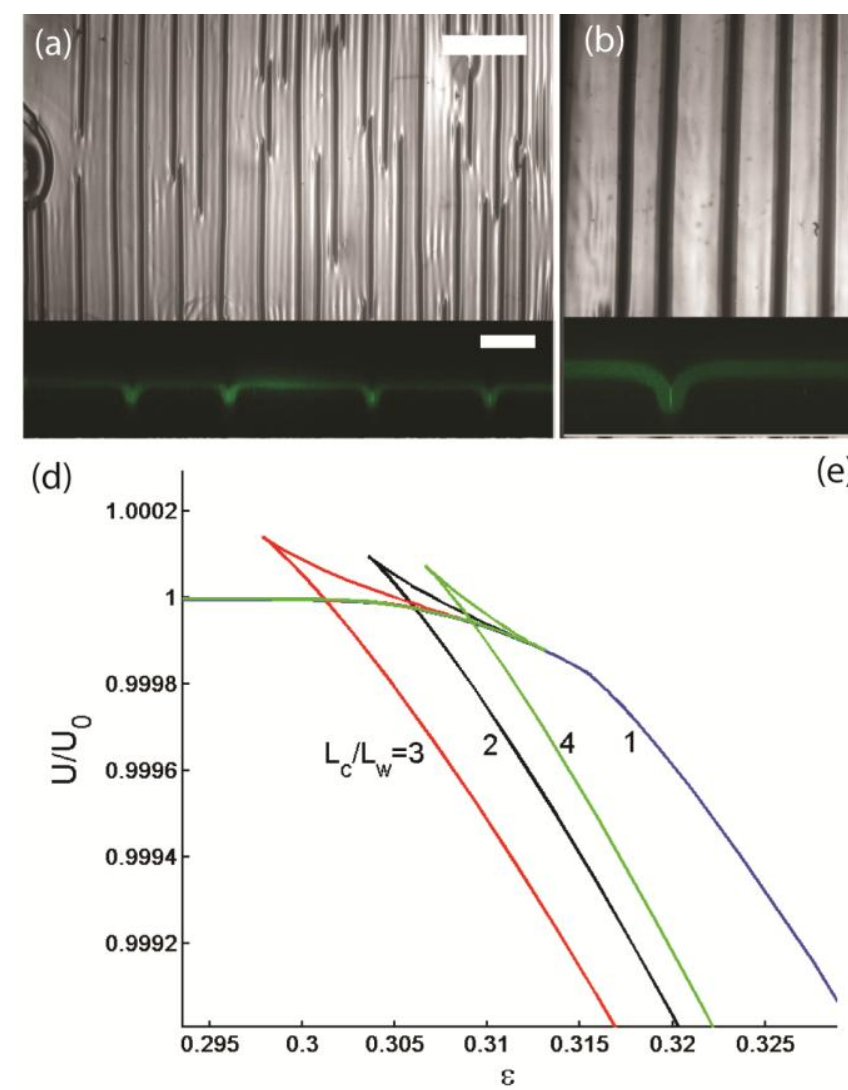
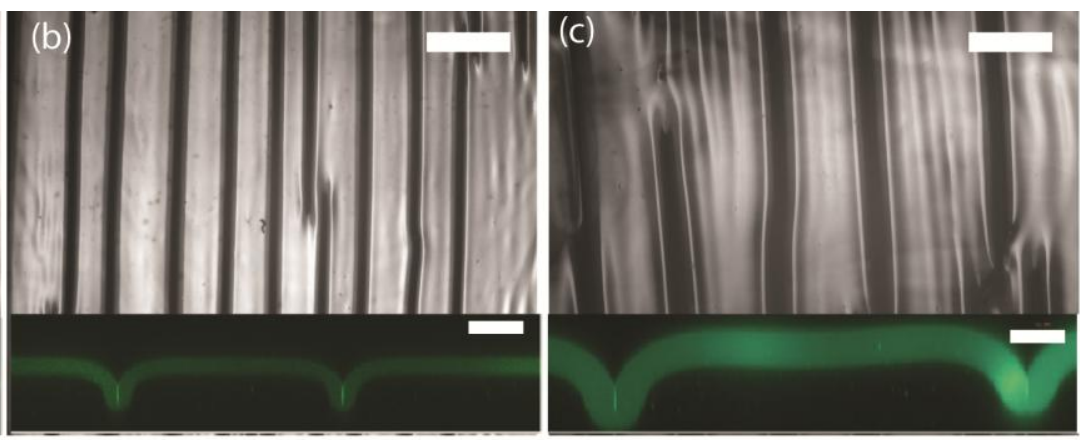

(e)

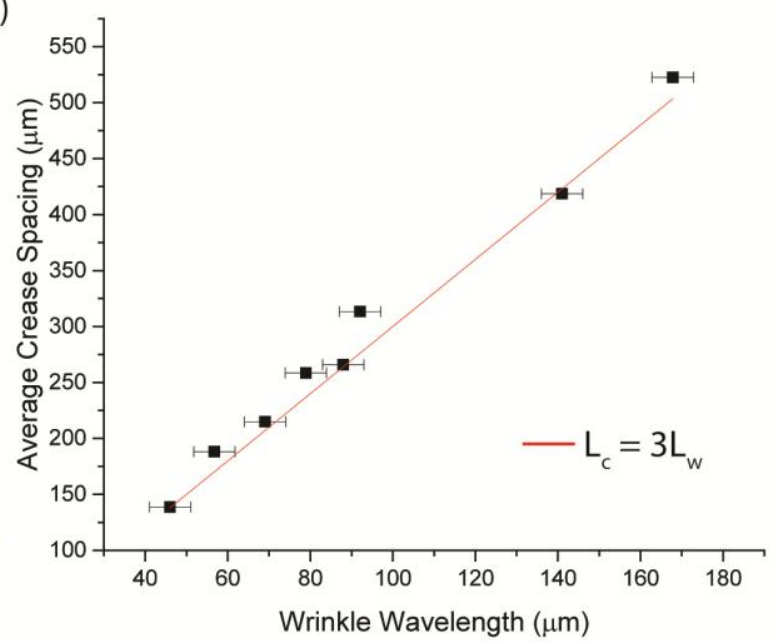

Fig. 4. (a, b, c) Top view optical micrographs (top, $500 \mu \mathrm{m}$ scale bar) and cross-sectional 
confocal images (bottom, $50 \mu \mathrm{m}$ scale bar) for creases formed by type 1 post-wrinkle bifurcation, where the film thickness increases from (a) $6 \pm 1 \mu \mathrm{m}$ (at $\varepsilon=0.26$ ), to (b) $17 \pm 1 \mu \mathrm{m}$ (at $\varepsilon=0.30$ ), and to (c) $26 \pm 2 \mu \mathrm{m}$ (at $\varepsilon=0.29$ ), while the thickness of the substrate remains the same $(400 \mu \mathrm{m})$. The film thickness affects crease spacing; the crease spacing for (a) is $140 \mu \mathrm{m}$, for (b) is $265 \mu \mathrm{m}$, and for (c) is $523 \mu \mathrm{m}$. (d) Simulation results of the bifurcation diagram with strain $\varepsilon$ as the loading parameter and the free energy as the state of the field for $G_{f} / G_{s}=3$. Different curves are for different spacing of creases. In the strain range covered by the simulation $0 \leq \varepsilon \leq 0.47$, the spacing of creases as 3 times of wrinkle wavelength has the lowest energy. (e) Experimental result of the average crease spacing as a function of wrinkle wavelength.

As a further demonstration that creases formed by type 1 post-wrinkle bifurcation differ from creases in the substrate alone, we characterize the effect of film thickness on crease spacing. Creases in a thick soft substrate are known[32] to adopt a spacing of approximately $3.5(1-\varepsilon) \mathrm{H}_{\mathrm{s}}$; however, we find in bilayers that the crease spacing in type 1 post-wrinkle bifurcations is controlled by the film thickness rather than the substrate thickness (Fig. 4). Example optical micrographs (top views) of creases formed by type 1 post-wrinkle bifurcation, along with corresponding confocal cross-sections $(50 \mu \mathrm{m}$ scale bar) are shown for film thicknesses of $6 \pm 1,17 \pm 1$, and $26 \pm 2 \mu \mathrm{m}$, at constant substrate thickness $(400 \mu \mathrm{m})$. The average spacing between creases $L_{\mathrm{c}}$ can clearly be seen to increase with film thickness, with respective values of 140,265 , and $523 \mu \mathrm{m}$. Here, we measure $L_{\mathrm{c}}$ by drawing a line along the compression direction across each top view micrograph, and dividing the length of the line by the number of creases intersected by the line.

To better understand the relationship between the crease spacing and the wrinkle wavelength, we performed finite element simulations with different box sizes, where we ensure that several wrinkles transition to one crease by prescribing only one geometric defect to initiate the crease. Therefore, we can vary the crease spacing by varying the size of the simulation box. Fig. $4 \mathrm{~d}$ shows the free energy $U / U_{0}$ as a function of strain $\varepsilon$ for $G_{f} / G_{s}=3$ with different crease spacings $L_{c} / L_{w}=1,2,3,4$. The free energy of the creased state for $L_{c} / L_{w}=3$ becomes lower than the wrinkled state earlier than for other spacings. Moreover, in the range of strain covered by our simulation $0 \leq \varepsilon \leq 0.47$, the mode $L_{c} / L_{w}=3$ always has the lowest energy, which is different than the value of $L_{\mathrm{c}} / L_{\mathrm{w}}=4$ predicted by Kim and co-workers[39]. As shown in Fig. 
$4 \mathrm{e}$, our prediction is in excellent agreement with the experimental measurements (where the wrinkle wavelength $L_{\mathrm{w}}$ is measured at the strain where creases first appear).

\section{Type 2 post-wrinkle bifurcation}

For a modulus ratio between 5.86 and 13.89, Kim and coworkers predicted that wrinkles will first undergo period doubling, followed by formation of creases[39]. We call this a 'type 2' post-wrinkle bifurcation. We analyze the type 2 post-wrinkle bifurcation through finite element simulations for an elastic bilayer with modulus ratio $G_{f} / G_{s}=8$ under uniaxial compression. We model one unit of period doubling. Making use of the mirror symmetry condition, we control the size of the simulation box to be one wrinkle wavelength. We introduce the defect so that a wrinkle trough forms on the symmetric boundary, grows deeper during period doubling, and then forms a crease. Therefore, one crease forms in every two wrinkles. Fig. 5a shows the simulation result of the bifurcation diagram with the strain $\varepsilon$ as the loading parameter, and the energy normalized by that of the flat state $U / U_{0}$ as the state of the field. The flat state is the only solution before the critical strain for the initiation of wrinkles $\varepsilon=0.174$. With the further increase of the strain, wrinkles form and the energy becomes lower than the homogeneous state. At $\varepsilon=0.202$, the period doubled state occurs and further decreases the energy compared to the wrinkled state. Soon after that, creases form on the lower trough in the period doubled state. Fig. $5 \mathrm{~b}$ is the bifurcation diagram with the amplitude normalized by the film thickness $H_{f}$ as the state of the field. The curves for the two different types of troughs remain at zero in the flat state, become non-zero after the wrinkles form, and bifurcate from each other after period doubling. The amplitude for the crease tip increases with further increases of the strain, while the amplitude for the other type of wrinkle trough, where a crease does not form, decreases. 
(a)

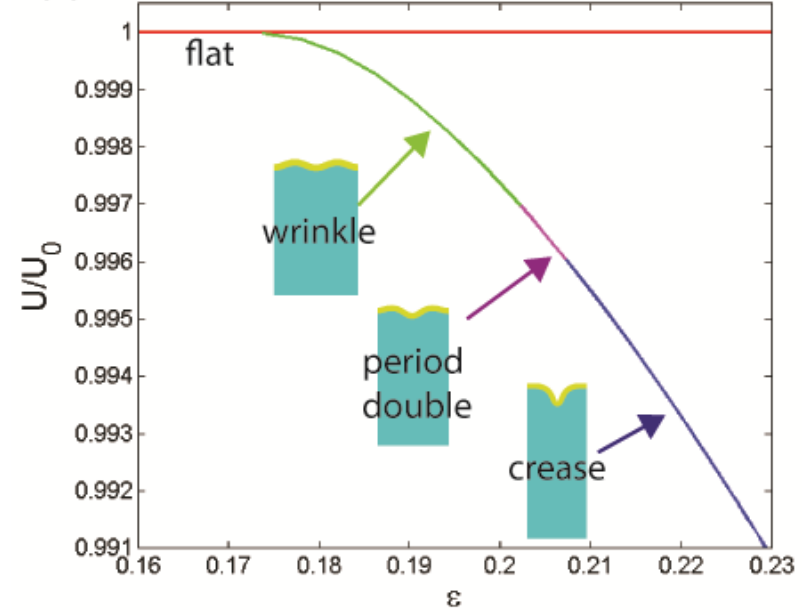

(b)

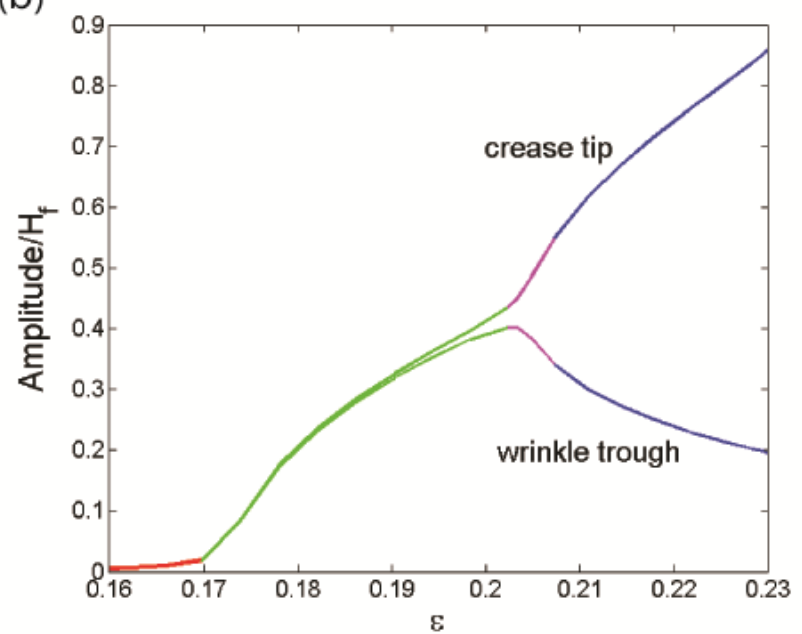

Fig. 5. Type 2 post-wrinkle bifurcation: wrinkles first undergo period doubling and then transition to creases $\left(G_{f} / G_{s}=8\right)$. In both bifurcation diagrams, the loading parameter is the uniaxial strain $\varepsilon$. (a) The state of the field is represented by the free energy of the block normalized by the flat state $U / U_{0}$. The insets show the simulation results of the wrinkle, period doubling and the crease states. (b) The amplitude between the wrinkle trough and peak normalized by the film thickness $H_{f}$ is chosen as the state of the field.

The experimental results for the type 2 post-wrinkle bifurcation show a transition of wrinkles to creases that closely match these simulations. The loading and unloading of a bilayer with a modulus ratio of approximately $5(0.4 \pm 0.05 \mathrm{MPa}$ substrate and $2.1 \pm 0.5 \mathrm{MPa}$ film $)$ is shown in Fig. 6a. The surface remains in the flat state until wrinkles form at a strain of about 0.15. Afterwards, wrinkles grow in amplitude with further increases in the strain. At a strain of 0.28, the wrinkles undergo period doubling - i.e., every other wrinkle grows in amplitude, while the others diminish. However, unlike the traditional period doubled state in bilayers with high modulus contrast, creases form in the troughs of the deeper wrinkles, as can be seen from the shift from a smooth ' $U$ ' shape, to a ' $V$ ' shape in every other trough. As compression increases, the amplitude of the self-contacting crease increases, while the amplitudes of the neighboring wrinkle troughs continue to diminish. Upon subsequently unloading, the self-contacting depth of the crease decreases while the amplitude of the other type of wrinkle trough increases. At a film strain of 0.25 , the shape of the crease tips returns to a ' $U$ ' shape but the bilayer still shows slight differences in amplitude between successive features (i.e., corresponding to the period doubled state). Upon further release of compression, the system returns to a wrinkled state and then 
eventually a flat state, highlighting that the type 2 post-wrinkle bifurcation is supercritical and has little to no hysteresis.

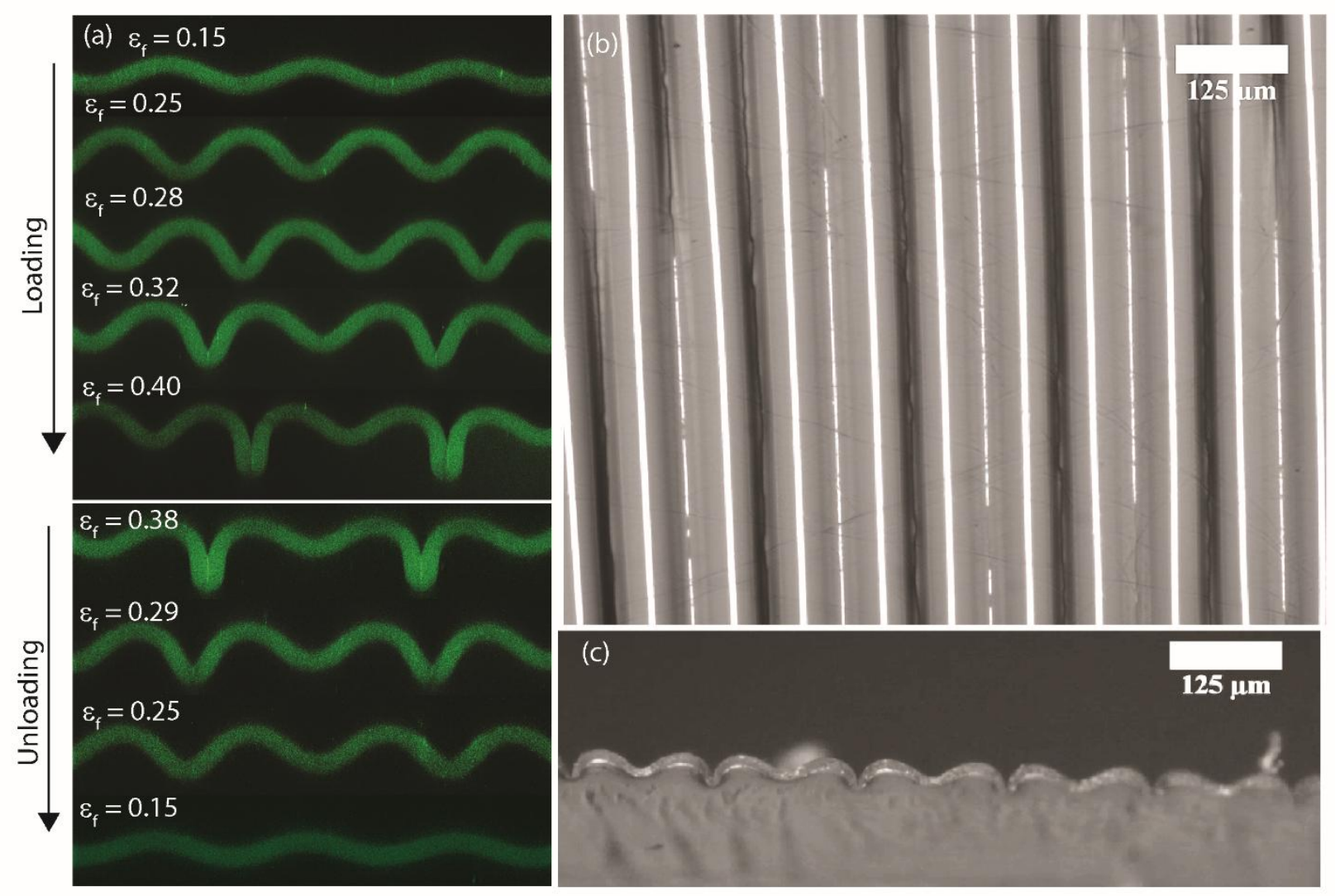

Fig. 6. Experimental observations of type 2 post-wrinkle bifurcation. (a) confocal image showing loading and unloading; optical micrographs showing (b) top and (c) side views of a bilayer at $\varepsilon=0.26$ in the period doubled state with creases in the deeper troughs.

The top view optical micrograph of a bilayer having undergone type 2 post-wrinkle bifurcation with a modulus contrast of approximately 5 in Fig. $6 \mathrm{~b}$ shows uniform creases, characterized by the dark lines, with a spacing equal to two times the wrinkle wavelength. The thick solid white lines are the peaks of the wrinkles while the thinner solid white lines are the shallow wrinkle troughs. The cross-sectional optical micrograph in Fig. 6c shows that creases form in every other wrinkle trough, and that these troughs have a qualitatively different shape than those without creases. Since the crease spacing is dictated by the location of the deeper trough in the period doubled state, creases adopt a highly uniform spacing of 2 times the wrinkle wavelength (ignoring the influence of defects in the initial wrinkle pattern). This result is 
different from the predicted crease spacing of 4 times the wrinkle wavelength of a type 2 postwrinkle bifurcation by Kim and co-workers[39].

\section{Conclusion}

For elastic bilayers with a ratio of the elastic moduli of the film to the substrate between 2 and 10, we have experimentally and computationally characterized post-wrinkle bifurcation of two types. In type 1 post-wrinkle bifurcation, wrinkles transition into creases by a subcritical instability, without the appearance of period-doubling. In type 2 post-wrinkle bifurcation, wrinkles first undergo period doubling, followed by supercritical formation of creases. For type 1 post-wrinkle bifurcation, the crease spacing is dictated by the film thickness and is, on average, three times the wrinkle wavelength, although creases do not form a regularly spaced array. For type 2 post-wrinkle bifurcation, creases form in each of the deeper troughs in the period doubled state, thereby providing a regular pattern of creases with spacing equal to twice the wrinkle wavelength. We anticipate that the understanding gained of surface instabilities within elastic bilayers of modest modulus contrast may have relevance for pattern formation in a variety of biological and engineering systems.

\section{Acknowledgments}

The work at UMass was supported by the National Science Foundation through grant NSF

DMR-130931, with additional support for AA through an NSF Graduate Research Fellowship (DGE-0907995). The work at Harvard was supported by the NSF MRSEC (DMR-1420570).

\section{References}

[1] J.A. Rogers, T. Someya, Y. Huang, Materials and mechanics for stretchable electronics., Science. 327 (2010) 1603-7. doi:10.1126/science.1182383.

[2] D.-Y. Khang, H. Jiang, Y. Huang, J.A. Rogers, A stretchable form of single-crystal silicon for high-performance electronics on rubber substrates., Science. 311 (2006) 208-12. doi:10.1126/science.1121401. 
[3] B. Xu, D. Chen, R.C. Hayward, Mechanically Gated Electrical Switches by Creasing of Patterned Metal/Elastomer Bilayer Films, Adv. Mater. 26 (2014) 4381-4385.

doi:10.1002/adma.201400992.

[4] J.B. Kim, P. Kim, N.C. Pegard, S.J. Oh, C.R. Kagan, J.W. Fleischer, H.A. Stone, Y. Loo, Wrinkles and deep folds as photonic structures in photovoltaics, Nat. Photonics. 6 (2012) 327. doi:10.1038/NPHOTON.2012.70.

[5] J. Kim, J. Yoon, R.C. Hayward, Dynamic display of biomolecular patterns through an elastic creasing instability of stimuli-responsive hydrogels., Nat. Mater. 9 (2010) 159-64. doi:10.1038/nmat2606.

[6] M. Arifuzzaman, Z.L. Wu, R. Takahashi, T. Kurokawa, T. Nakajima, J.P. Gong, Geometric and edge effects on swelling-induced ordered structure formation in polyelectrolyte hydrogels, Macromolecules. 46 (2013) 9083-9090. doi:10.1021/ma401773w.

[7] A. Schweikart, A. Fery, Controlled wrinkling as a novel method for the fabrication of patterned surfaces, (2009) 249-263. doi:10.1007/s00604-009-0153-3.

[8] Q. Li, X. Han, J. Hou, J. Yin, S. Jiang, C. Lu, Patterning Poly(dimethylsiloxane) Microspheres via Combination of Oxygen Plasma Exposure and Solvent Treatment, J. Phys. Chem. B. 119 (2015) 13450-13461. doi:10.1021/acs.jpcb.5b07208.

[9] S.G. Lee, D.Y. Lee, H.S. Lim, D.H. Lee, S. Lee, K. Cho, Switchable transparency and wetting of elastomeric smart windows., Adv. Mater. 22 (2010) 5013-7.

doi:10.1002/adma.201002320.

[10] P. Görrn, M. Lehnhardt, W. Kowalsky, T. Riedl, S. Wagner, Elastically tunable selforganized organic lasers., Adv. Mater. 23 (2011) 869-72. doi:10.1002/adma.201003108.

[11] C. Harrison, C.M. Stafford, W. Zhang, A. Karim, Sinusoidal phase grating created by a tunably buckled surface, Appl. Phys. Lett. 85 (2004) 4016. doi:10.1063/1.1809281.

[12] E. Lee, M. Zhang, Y. Cho, Y. Cui, J. Van der Spiegel, N. Engheta, S. Yang, Tilted pillars on wrinkled elastomers as a reversibly tunable optical window., Adv. Mater. 26 (2014) 4127-33. doi:10.1002/adma.201400711.

[13] P.-C. Lin, S. Vajpayee, A. Jagota, C.-Y. Hui, S. Yang, Mechanically tunable dry adhesive from wrinkled elastomers, Soft Matter. 4 (2008) 1830. doi:10.1039/b802848f.

[14] E.P. Chan, E.J. Smith, R.C. Hayward, A.J. Crosby, Surface Wrinkles for Smart Adhesion, Adv. Mater. 20 (2008) 711-716. doi:10.1002/adma.200701530.

[15] E.P. Chan, J.M. Karp, R.S. Langer, A "self-pinning" adhesive based on responsive surface wrinkles, J. Polym. Sci. Part B Polym. Phys. 49 (2011) 40-44. doi:10.1002/polb.22165.

[16] J.Y. Chung, J.P. Youngblood, C.M. Stafford, Anisotropic wetting on tunable microwrinkled surfaces, Soft Matter. 3 (2007) 1163. doi:10.1039/b705112c.

[17] K. Khare, J. Zhou, S. Yang, Tunable open-channel microfluidics on soft poly(dimethylsiloxane) (PDMS) substrates with sinusoidal grooves., Langmuir. 25 (2009) 12794-9. doi:10.1021/la901736n.

[18] J. Dervaux, M. Ben Amar, Morphogenesis of Growing Soft Tissues, Phys. Rev. Lett. 101 (2008) 068101. doi:10.1103/PhysRevLett.101.068101. 
[19] C.T. McKee, J.A. Last, P. Russell, C.J. Murphy, Indentation versus Tensile Mesurements of Young ’s Modulus for Soft Biological Tissues, Tissue Eng. B. 17 (2011).

doi:10.1089/ten.teb.2010.0520.

[20] J. Yin, X. Chen, I. Sheinman, Anisotropic buckling patterns in spheroidal film/substrate systems and their implications in some natural and biological systems, J. Mech. Phys. Solids. 57 (2009) 1470-1484. doi:10.1016/j.jmps.2009.06.002.

[21] B.R. Wiggs, C.A. Hrousis, J.M. Drazen, R.D. Kamm, On the mechanism of mucosal folding in normal and asthmatic airways., J. Appl. Physiol. 83 (1997) 1814-1821.

[22] T. Tallinen, J.Y. Chung, J.S. Biggins, L. Mahadevan, Gyrification from constrained cortical expansion., Proc. Natl. Acad. Sci. U. S. A. 111 (2014) 12667-72. doi:10.1073/pnas.1406015111.

[23] W. Yang, T.C. Fung, K.S. Chian, C.K. Chong, Instability of the two-layered thick-walled esophageal model under the external pressure and circular outer boundary condition, J. Biomech. 40 (2007) 481-490. doi:10.1016/j.jbiomech.2006.02.020.

[24] J.-Y. Sun, S. Xia, M.-W. Moon, K.H. Oh, K.-S. Kim, Folding wrinkles of a thin stiff layer on a soft substrate, Proc. R. Soc. A Math. Phys. Eng. Sci. 468 (2011) 932-953. doi:10.1098/rspa.2011.0567.

[25] J. Genzer, J. Groenewold, Soft matter with hard skin: From skin wrinkles to templating and material characterization, Soft Matter. 2 (2006) 310. doi:10.1039/b516741h.

[26] Z.Y. Huang, W. Hong, Z. Suo, Nonlinear analyses of wrinkles in a film bonded to a compliant substrate, J. Mech. Phys. Solids. 53 (2005) 2101-2118. doi:10.1016/j.jmps.2005.03.007.

[27] L. Jin, A. Auguste, R.C. Hayward, Z. Suo, Bifurcation diagrams for the formation of wrinkles or creases in soft bilayers, J. Appl. Mech. 82 (2015) 1-11. doi:10.1115/1.4030384.

[28] Q. Wang, X. Zhao, Phase Diagrams of Instabilities in Compressed Film-Substrate Systems, J. Appl. Mech. 81 (2013). doi:10.1115/1.4025828.

[29] J. Yoon, J. Kim, R.C. Hayward, Nucleation, growth, and hysteresis of surface creases on swelled polymer gels, Soft Matter. 6 (2010) 5807. doi:10.1039/c0sm00372g.

[30] W. Hong, X. Zhao, Z. Suo, Formation of creases on the surfaces of elastomers and gels, Appl. Phys. Lett. 95 (2009) 111901. doi:10.1063/1.3211917.

[31] E. Hohlfeld, L. Mahadevan, Unfolding the Sulcus, Phys. Rev. Lett. 106 (2011) 105702. doi:10.1103/PhysRevLett.106.105702.

[32] S. Cai, D. Chen, Z. Suo, R.C. Hayward, Creasing instability of elastomer films, Soft Matter. 8 (2012) 1301. doi:10.1039/c2sm06844c.

[33] F. Brau, P. Damman, H. Diamant, T.A. Witten, Wrinkle to fold transition: influence of the substrate response, Soft Matter. 9 (2013) 8177. doi:10.1039/c3sm50655j.

[34] F. Brau, H. Vandeparre, A. Sabbah, C. Poulard, A. Boudaoud, P. Damman, Multiplelength-scale elastic instability mimics parametric resonance of nonlinear oscillators, Nat. Phys. 7 (2010) 56-60. doi:10.1038/nphys1806.

[35] L. Pocivavsek, R. Dellsy, A. Kern, S. Johnson, B. Lin, K.Y.C. Lee, E. Cerda, Stress and 
fold localization in thin elastic membranes., Science. 320 (2008) 912-6. doi:10.1126/science.1154069.

[36] J.W. Hutchinson, The role of nonlinear substrate elasticity in the wrinkling of thin films, Philos. Trans. R. Soc. A Math. Phys. Eng. Sci. 371 (2013).

[37] A. Auguste, L. Jin, Z. Suo, R.C. Hayward, The role of substrate pre-stretch in postwrinkling bifurcations., Soft Matter. 10 (2014) 6520-9. doi:10.1039/c4sm01038h.

[38] Y.B. Fu, P. Ciarletta, Buckling of a coated elastic half-space when the coating and substrate have similar material properties, Proc. R. Soc. A Math. Phys. Eng. Sci. 471 (2015) 20140979.

[39] R. Zhao, T. Zhang, M. Diab, H. Gao, K.-S. Kim, The primary bilayer ruga-phase diagram I: Localizations in ruga evolution, Extrem. Mech. Lett. 4 (2015) 76-82. doi:10.1016/j.eml.2015.04.006.

[40] Y. Cao, J.W. Hutchinson, Wrinkling Phenomena in Neo-Hookean Film/Substrate Bilayers, J. Appl. Mech. 79 (2012) 031019. doi:10.1115/1.4005960.

[41] Q. Wang, X. Zhao, A three-dimensional phase diagram of growth-induced surface instabilities, Sci. Rep. 5 (2015) 8887. doi:10.1038/srep08887.

[42] T. Tallinen, J.S. Biggins, Mechanics of invagination and folding: Hybridized instabilities when one soft tissue grows on another, Phys. Rev. E - Stat. Nonlinear, Soft Matter Phys. 92 (2015) 1-8. doi:10.1103/PhysRevE.92.022720.

[43] M. Diab, T. Zhang, R. Zhao, H. Gao, K. Kim, P.R.S. A, Ruga mechanics of creasing: from instantaneous to setback creases Ruga mechanics of creasing: from instantaneous to setback creases, Proc. R. Soc. A Math. Phys. Eng. Sci. 469 (2013) 20120753.

[44] Y.-C. Chen, A.J. Crosby, High Aspect Ratio Wrinkles via Substrate Prestretch, Adv. Mater. 26 (2014) 5626-5631. doi:10.1002/adma.201401444.

[45] A. Takei, L. Jin, J.W. Hutchinson, H. Fujita, Ridge Localizations and Networks in Thin Films Compressed by the Incremental Release of a Large Equi-biaxial Pre-stretch in the Substrate., Adv. Mater. (2014) 1-7. doi:10.1002/adma.201306162.

[46] D. Chen, L. Jin, Z. Suo, R.C. Hayward, Controlled formation and disappearance of creases, Mater. Horizons. 1 (2014) 207. doi:10.1039/c3mh00107e.

[47] L. Jin, D. Chen, R.C. Hayward, Z. Suo, Creases on the interface between two soft materials., Soft Matter. 10 (2014) 303-11. doi:10.1039/c3sm51512e.

[48] G. Miquelard-Garnier, A.B. Croll, C.S. Davis, A.J. Crosby, Contact-line mechanics for pattern control, Soft Matter. 6 (2010) 5789. doi:10.1039/c0sm00165a.

[49] S. Park, Y.S. Huh, H.G. Craighead, D. Erickson, A method for nanofluidic device prototyping using elastomeric collapse., Proc. Natl. Acad. Sci. U. S. A. 106 (2009) 1554915554. doi:10.1073/pnas.0904004106. 